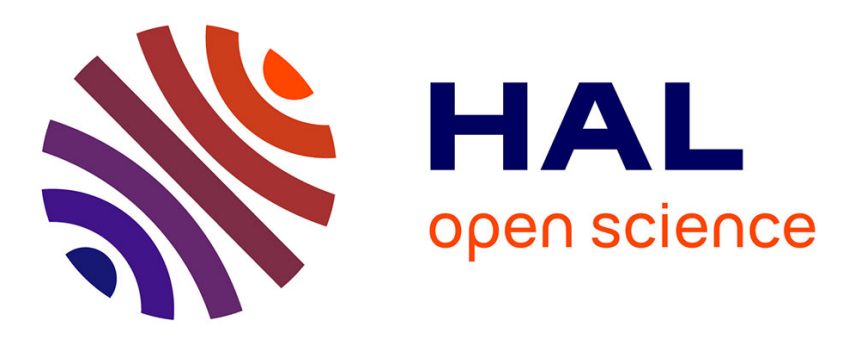

\title{
Mesure assistée par miniordinateur du pouvoir thermoélectrique de petits échantillons
}

\author{
A. Bonnet, P. Said, A. Conan
}

\section{To cite this version:}

A. Bonnet, P. Said, A. Conan. Mesure assistée par miniordinateur du pouvoir thermoélectrique de petits échantillons. Revue de Physique Appliquée, 1982, 17 (10), pp.701-706. 10.1051/rphysap:019820017010070100 . jpa-00245048

\section{HAL Id: jpa-00245048 https://hal.science/jpa-00245048}

Submitted on 1 Jan 1982

HAL is a multi-disciplinary open access archive for the deposit and dissemination of scientific research documents, whether they are published or not. The documents may come from teaching and research institutions in France or abroad, or from public or private research centers.
L'archive ouverte pluridisciplinaire HAL, est destinée au dépôt et à la diffusion de documents scientifiques de niveau recherche, publiés ou non, émanant des établissements d'enseignement et de recherche français ou étrangers, des laboratoires publics ou privés. 
Classification

Physics Abstracts

07.20

\title{
Mesure assistée par miniordinateur du pouvoir thermoélectrique de petits échantillons
}

\author{
A. Bonnet, P. Said et A. Conan \\ Laboratoire de Physique des Matériaux et Composants de l'Electronique, \\ 2, rue de la Houssinière, 44072 Nantes Cedex, France
}

(Reçu le 1er octobre 1981, révisé le 17 mai 1982, accepté le 25 mai 1982)

\begin{abstract}
Résumé. - Le dispositif décrit permet une mesure du pouvoir thermoélectrique d'échantillons de faible dimension (quelques $\mathrm{mm}$ ) entre 4 et $800 \mathrm{~K}$. La mesure est assistée par une chaîne d'acquisition de données sous le contrôle d'un calculateur. Il est possible de mesurer le P.T.E. d'échantillons métalliques $(S<10 \mu \mathrm{V} / \mathrm{K})$ comme le P.T.E. d'échantillons semi-conducteurs à fort coefficient de Seebeck $(S>500 \mu \mathrm{V} / \mathrm{K})$ avec une précision de $2 \%$. L'appareil a été testé sur un échantillon de fer électrolytique standard.
\end{abstract}

\begin{abstract}
A method is described for measurements of the thermoelectric power of small samples in the temperature range 4-800 $\mathrm{K}$. The help of a data acquisition system monitored by a calculator is required. Measurements may be performed on metallic samples $(S<10 \mu \mathrm{V} / \mathrm{K})$ as well as on semiconductors with high Seebeck coefficient $(S>500 \mu \mathrm{V} / \mathrm{K})$ within an accuracy of $2 \%$. The device has been checked on a standard electrolytic sample.
\end{abstract}

1. Introduction. - Les mécanismes de conduction dans les solides ont été souvent explicités par la mesure de la variation du pouvoir thermoélectrique $S$ et de la conductivité électrique en fonction de la température $[1,2]$.

La détermination du pouvoir thermoélectrique implique une mesure de la différence de température $\Delta T$ existant aux bornes de l'échantillon étudié et de la f.é.m. correspondante $\Delta V$. La mesure des f.é.m. thermoélectriques devant être effectuée en circuit ouvert, le système de mesure doit présenter une impédance d'entrée très supérieure à celle des échantillons, sans toutefois dépasser une certaine limite au-dessus de laquelle le niveau de bruit serait plus important et conduirait à choisir un $\Delta T$ plus élevé. L'élimination des potentiels de contact, des f.é.m. thermoélectriques parasites (effets Peltier locaux dûs à des contraintes sur les fils de thermocouple) impose le relevé d'au moins deux points de mesure $\left(\left\{\Delta V_{1}, \Delta T_{1}\right\},\left\{\Delta V_{2}\right.\right.$, $\left.\left.\Delta T_{2}\right\}\right)$.

La méthode dynamique $[3,4,5]$, caractérisée par l'évolution contrôlée ou la modulation du gradient thermique présente l'inconvénient de maintenir un retard systématique, variable en fonction du temps, dépendant de la conductivité thermique des fils de chacun des thermocouples, entre l'excitation thermique et la tension résultante : idéalement, ce retard devrait être estimé et les corrections effectuées pendant toute la durée de la mesure du pouvoir thermoélectrique à une température donnée.

Pour éviter ces difficultés, la méthode statique [6] a été retenue : elle nécessite l'obtention d'au moins deux paliers de température pour lesquels sont mesurées les f.é.m. thermoélectriques; la mesure sur un troisième palier permet le contrôle éventuel de la stabilité des f.é.m. parasites. Les températures moyennes de l'échantillon, mesurées sur chacun des 2 paliers $\left(\bar{T}_{1}\right.$ et $\left.\bar{T}_{2}\right)$, peuvent présenter une légère différence de l'ordre du $1 / 2^{\circ} \mathrm{C}$ qui entraîne une erreur systématique sur la mesure du P.T.E. $S(T)$ attribuée à la température $\bar{T}=\frac{\bar{T}_{1}+\bar{T}_{2}}{2}$.

Dans le cas simple d'une variation quadratique de la f.é.m. de Seebeck, on est conduit à la relation suivante qui reste valable dans le cas général, au second ordre près, et dans la mesure où $\mathrm{d} S / \mathrm{d} T$ reste finie :

$$
\begin{aligned}
S_{\text {mesurée }}=\frac{\Delta V_{1}-\Delta V_{2}}{\Delta T_{1}-\Delta T_{2}}= & S(\bar{T})+ \\
& +\left(\frac{\mathrm{d} S}{\mathrm{~d} T}\right)_{\bar{T}} \cdot \theta \cdot \frac{\Delta T_{1}+\Delta T_{2}}{\Delta T_{1}-\Delta T_{2}}
\end{aligned}
$$

où $\theta$ est la variation de température moyenne entre les 
deux paliers et où les indices 1 et 2 se rapportent au premier et au deuxième paliers. La condition $\theta=0$ qui suppose le contrôle parfait de la régulation est difficile à réaliser pratiquement. Il est plus facile d'obtenir des différences de température symétriques : $\Delta T_{1}=-\Delta T_{2}$.

La méthode à 4 fils [4] requiert la mesure des f.é.m. thermoélectriques $\Delta V$ (métal A-échantillon S-métal A) et $\Delta U$ (métal B-échantillon S-métal B) pour un même $\Delta T$ (Fig. 1). Elle conduit à l'expression suivante du

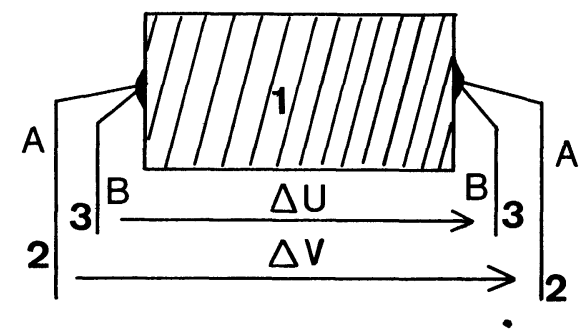

Fig. 1. - Mesure des f.é.m. thermoélectriques par la méthode à 4 fils.

[Measurement of thermoelectric e.m.f. by the 4-wires method.]

pouvoir thermoélectrique de l'échantillon par rapport au métal A :

$$
\begin{aligned}
S_{\mathrm{S} / \mathbf{A}}(\bar{T})=\frac{\Delta V_{1}-\Delta V_{2}}{\left(\Delta V_{1}-\Delta V_{2}\right)-\left(\Delta U_{1}-\Delta U_{2}\right)} \times \\
\times S_{\mathbf{B} / \mathbf{A}}(\bar{T})=\frac{E_{\mathbf{A}}}{E_{\mathbf{A}}-E_{\mathbf{B}}} S_{\mathbf{B} / \mathbf{A}}(\bar{T})
\end{aligned}
$$

où

$$
\begin{gathered}
\bar{T}=\frac{\bar{T}_{1}+\bar{T}_{2}}{2}, \\
E_{\mathrm{A}}=\Delta V_{1}-\Delta V_{2}, \quad E_{\mathrm{B}}=\Delta U_{1}-\Delta U_{2} .
\end{gathered}
$$

L'erreur relative sur le P.T.E. s'écrit :

$$
\frac{\Delta S}{S}=\Delta E \cdot \frac{\left|E_{\mathrm{A}}\right|+\left|E_{\mathrm{B}}\right|}{\left|E_{\mathrm{A}} \cdot\left(E_{\mathrm{A}}-E_{\mathrm{B}}\right)\right|}+\frac{\Delta S_{\mathrm{B} / \mathrm{A}}}{S_{\mathrm{B} / \mathrm{A}}}
$$

où $\Delta E=\Delta E_{\mathrm{A}}=\Delta E_{\mathrm{B}}$.

Il est évident que l'exploration $\left|\Delta T_{1}-\Delta T_{2}\right|$ doit être relativement grande : en pratique, on retient $\left|E_{\mathrm{A}}-E_{\mathrm{B}}\right| \sim 10 \mu \mathrm{V}$ pour des fils de thermocouple $\mathrm{Cu}-\mathrm{Cst}$ au voisinage de la température ambiante.

Le couple de mesures $\{\Delta V, \Delta T\}$ risque d'être d'autant plus entaché d'erreur que les mesures de $\Delta U$ et $\Delta V$ ont lieu à des instants différents et que les valeurs de ces mêmes f.é.m. thermoélectriques sont grandes (P.T.E. élevé). En effet, dans le cas de la méthode statique, il existe toujours sur chaque palier des fluctuations des f.é.m. qui peuvent être de l'ordre de grandeur (si le P.T.E. est élevé) de celle qui définit $\Delta T$ : prenons le cas d'un P.T.E. de $500 \mu \mathrm{V} / \mathrm{K}$, une variation de $1 / 100^{e}$ de degré se traduit par une variation de f.é.m. de $5 \mu \mathrm{V}$ comparable avec un $\Delta T$ de $1 / 4^{\circ} \mathrm{C}$ à la température ambiante et pour un thermocouple $\mathrm{Cu}-\mathrm{Cst}$. Une solution consisterait à adopter la méthode différentielle statique, mais elle présenterait l'inconvénient d'introduire une résistance thermique entre l'une des faces de l'échantillon et la soudure du thermocouple monté en différentiel. Finalement, la méthode à 4 fils a été retenue en réalisant au mieux la simultanéité des mesures de $\Delta V$ et $\Delta U$ par l'utilisation de deux appareils de mesure. Ce dispositif est décrit par la suite.

2. Cellule de mesure. - Les dimensions des échantillons (monocristaux, disque ou barreaux de poudre fortement compactés) sont de l'ordre de quelques millimètres. L'échantillon étudié est fixé sur un porteéchantillon qui s'adapte au cryostat pour les basses températures $(4 \mathrm{~K}-300 \mathrm{~K})$ et qui se place dans une cellule isotherme pour les hautes températures $(300 \mathrm{~K}$ $800 \mathrm{~K}$ ). Dans les deux cas, la cellule de mesure est chemisée afin d'éviter les fuites thermiques, et placée sous vide. Dans le cas du montage « vertical », une face de l'échantillon est en contact thermique avec la face régulée. Un microfour classique [4] ou un dispositif de chauffage par fibre optique utilisé par d'autres auteurs [3] permet d'établir le gradient thermique. Cette disposition ne nécessite qu'un seul four supplémentaire. Elle présente l'inconvénient de ne pouvoir assurer l'inversion du gradient thermique que pour des températures largement supérieures à celle du liquide cryogénique.

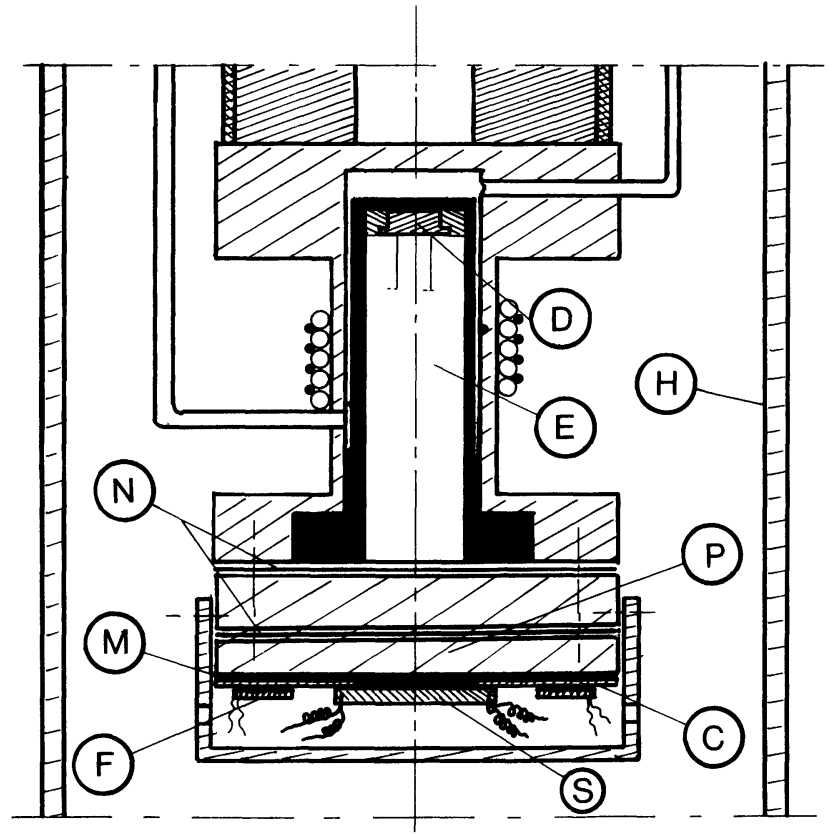

Fig. 2. - Cellule de mesure basse température : $\mathrm{C}$ : feuilles de clinquant d'or; D : diode As/Ga ; E : cheminée ; F : microfour ; $\mathbf{H}$ : queue du cryostat; $\mathbf{M}$ : pellicule isolante; $\mathbf{N}$ : galettes d'indium; $\mathrm{P}$ : porte-échantillon; $\mathrm{S}$ : échantillon.

[Low temperature sample chamber :C : gold sheet ; D:As-Ga diode ; E : chimney; $\mathbf{F}$ : micro-furnace ; $\mathbf{H}$ : cryostat ; $\mathbf{M}$ : insulator film; $\mathrm{N}$ : indium gasket $\mathrm{P}$ : sample holder; $\mathrm{S}$ : sample.] 
De plus, elle conduit à des fluctuations de température plus importantes sur les paliers de température. Le montage " horizontal " est caractérisé par le fait que l'échantillon repose à plat sur la face régulée, d'où la nécessité de prévoir deux microfours, destinés à établir le gradient thermique (Fig. 2). En raison des dissymétries inhérentes au montage, il s'établit naturellement un gradient thermique que l'on peut contrôler et même inverser dès les plus basses températures par action sur l'un ou l'autre des microfours (F). Cette disposition permet de travailler à gradient symétrique, la température moyenne est alors définie au second ordre près. Eventuellement, par réglage fin de la régulation, la température moyenne peut être maintenue égale pour les deux paliers de température, ce qui définit sans ambiguité cette dernière.

Les cellules de mesure basse et haute température sont représentées (Fig. 2 et Fig. 3) : une fine pellicule (M) isolante (araldite haute température) est déposée sur le porte-échantillon $(\mathrm{P})$. L'échantillon $(\mathrm{S})$ repose sur ses extrémités sur deux feuilles de clinquant d'or (C) et est lui-même collé et pressé sur le porte-échantillon.

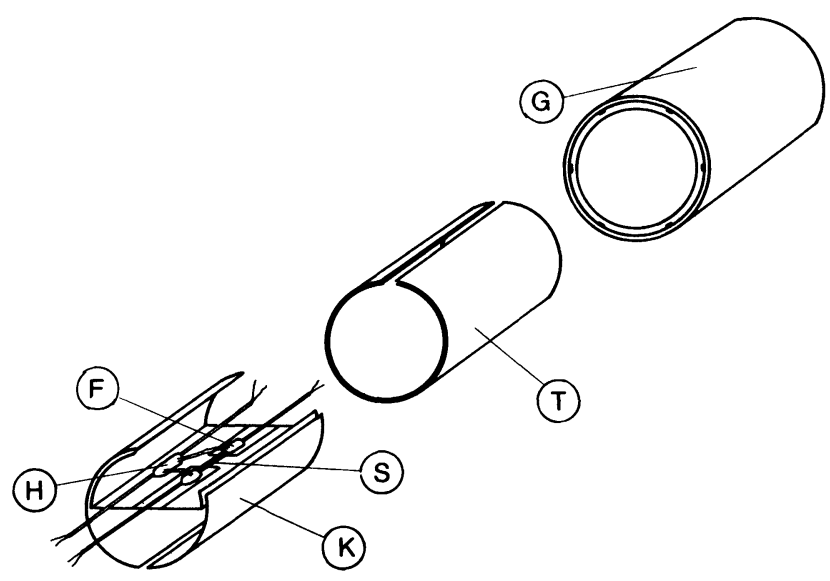

Fig. 3. - Cellule haute température : $\mathbf{K}$ : feuille d'aluminium; $\mathrm{T}$ : cylindre d'acier inoxydable; $\mathrm{F}$ : micro-four ; $\mathrm{G}$ : four principal ; $\mathrm{S}$ : échantillon; $\mathrm{H}$ : colle epoxy.

[High temperature sample chamber : $\mathrm{K}$ : aluminium sheet ; $\mathbf{T}$ : stainless steel cylinder $; \mathbf{F}:$ microfurnace; $\mathbf{G}$ : furnace; $\mathrm{S}$ : sample; $\mathrm{H}$ : epoxy bonding.]

Les micro-fours $(\mathrm{F})$ destinés à contrôler le gradient thermique doivent présenter l'inertie thermique la plus faible possible, aussi leurs dimensions sont-elles très inférieures à celles du porte-échantillon. Dans la cellule basse température, ils sont réalisés par un bobinage à plat de fil fin de constantan gainé téflon et ils reposent sur les feuilles de clinquant d'or. A haute température, il s'agit simplement de quelques tours de fil fin émaillé de constantan bobinés sur un petit mandrin d'alumine. Dans les deux cas, les microfours sont alimentés à tour de rôle à courant continu constant.

A basse température (Fig. 2), le contact thermique entre la queue du cryostat et le porte-échantillon est amélioré par interposition d'une mince galette d'indium $(\mathrm{N})$. Le capteur de température est une diode As-Ga (D) alimentée à courant constant, dont le boîtier est soudé à l'indium au fond de la cheminée (E). Le cryostat utilisé est un système classique à circulation de liquide cryogénique assurée par la dépression d'une pompe rotative. Pour les mesures limitées aux basses températures, des ancrages sont préparés par projection d'indium sous ultrasons aux extrémités de l'échantillon. Le thermocouple est réalisé par brasure à l'argent (haut point de fusion), puis la tête du thermocouple est soudée à l'indium (bas point de fusion) sur les ancrages préparés. Des volants thermiques constitués par des enroulements des fils de thermocouple autour de l'échantillon ou par contact de quelques spires de ces fils sur les feuilles de clinquant d'or (C) permettent de minimiser les fuites thermiques.

A haute température (Fig. 3) les deux extrémités de l'échantillon sont percées à $0,3 \mathrm{~mm}$ et reçoivent les têtes de thermocouple, le contact étant assuré par de la laque d'or. Cette technique permet d'effectuer des mesures dans tout le domaine de température $4 \mathrm{~K}$ $800 \mathrm{~K}$. L'échantillon (S) est placé dans une cellule constituée d'une feuille d'aluminium $(K)$, l'ensemble présentant une très faible inertie thermique. Cette cellule est insérée dans un cylindre fendu selon une génératrice et réalisé dans une tôle d'acier inoxydable (T). L'ensemble (K), (T) est introduit dans un four (G) régulé et mis sous vide, l'élasticité de $(\mathrm{T})$ assure un très bon contact. Les fils de thermocouple sont thermalisés dans quelques gouttes d'une colle époxy $(\mathrm{H})$ qui assure l'isolement électrique mais qui est très bonne conductrice thermique.

Dans les deux cas, le contact électrique des fils de thermocouple de l'échantillon et des fils de thermocouple de même nature et de même diamètre $(0,08 \mathrm{~mm}$ gainé téflon) en place dans la cellule de mesure est assuré par torsades. Celles-ci sont placées en contact thermique sur la queue du cryostat à basse température et sur le plateau de l'enceinte à vide à haute température. A l'extérieur, les soudures de référence (métal A-cuivre, métal B-cuivre) sont immergées dans un bain de pétrole à la température ambiante. Par rapport à la solution classique qui consiste à immerger les soudures dans la glace fondante, cette solution présente l'avantage d'un meilleur isolement électrique et d'une plus grande homogénéité de la température. La correction du $0^{\circ}$ est assurée par un thermocouple auxiliaire plongé dans la glace fondante.

3. Electronique de mesure. - Le schéma synoptique du systéme d'acquisition de données est représenté figure 4. Les données (f.é.m. thermoélectriques sont présentes sous forme de signaux analogiques sur les différentes voies du scanner H.P. 3495 A) qui sont sélectionnées au cours du déroulement du programme exécuté par le calculateur H.P. 9815. La f.é.m. $\Delta V$ (métal B-échantillon-métal B) est connectée directement et en permanence sur un nanovoltmètre KEITH- 


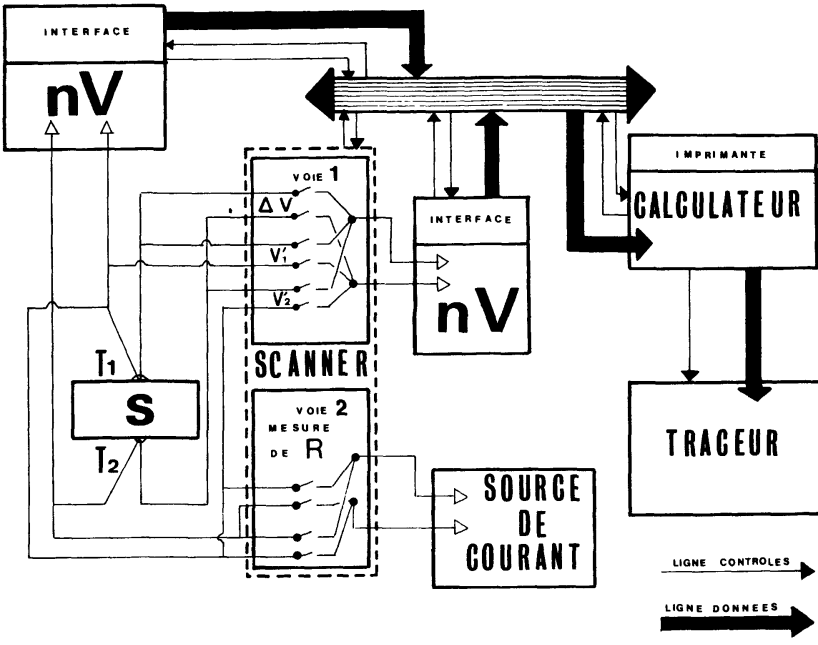

Fig. 4. - Système d'acquisition automatique de données.

[Data acquisition system.]

LEY 181. Les autres données sont mesurées par un deuxième nanovoltmètre, branché sur la sortie commune de la première décade du scanner. Cette disposition s'est révélée particulièrement intéressante et efficace lors de l'étude d'échantillons résistifs car elle permet de s'affranchir complètement des problèmes posés par le temps de montée de l'appareil de mesure. Les deux appareils effectuant des mesures tous les $3 / 10^{\mathrm{e}}$ de seconde, les f.é.m. $\Delta V$ et $\Delta U$ sont mesurées en alternance pendant environ $3 \mathrm{~s}$ (soit 10 couples de mesure) sans commutation des voies du scanner, ce qui permet d'éviter les erreurs dues aux feé.m. de contact du commutateur. Cette technique permet de moyenner l'erreur due à des défauts de corrélation (retards entre les thermocouples, ...) entre les couples de points de mesure et réalise au mieux la simultanéité des mesures de $\Delta U$ et $\Delta V$. La figure 6 représente le schéma électrique équivalent vu par le nanovolt-

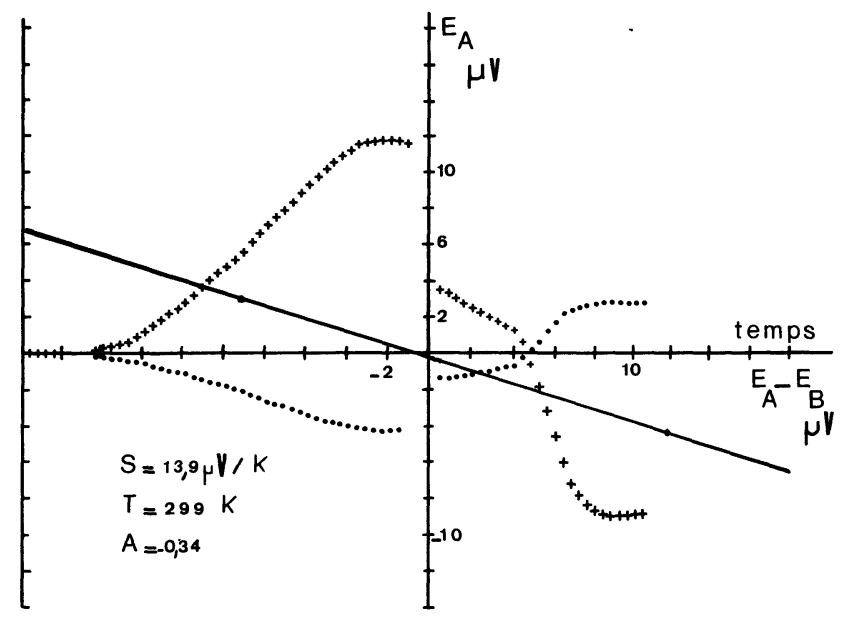

Fig. 5. - Enregistrement sur table traçante.

[Typical graphic plotter.] mètre pendant la mesure de la f.é.m. On est conduit à la relation approchée suivante :

$$
\begin{gathered}
\Delta u-\Delta v \frac{R}{R_{v}} \\
V_{\text {mesurée }} \sim\left(\Delta v-\Delta u \frac{R}{R_{u}}\right)
\end{gathered}
$$

avec $R_{v}, R_{u}$ impédances d'entrée des nanovoltmètres $\left(10^{9} \Omega\right.$ sur le calibre $\left.3 \mathrm{mV}\right)$,

$R$ impédance de l'échantillon $\left(R \ll R_{u}, R_{v}\right)$,

$R_{1}, R_{2}, R_{1}^{\prime}, R_{2}^{\prime}$ impédances des fils de thermocouples.

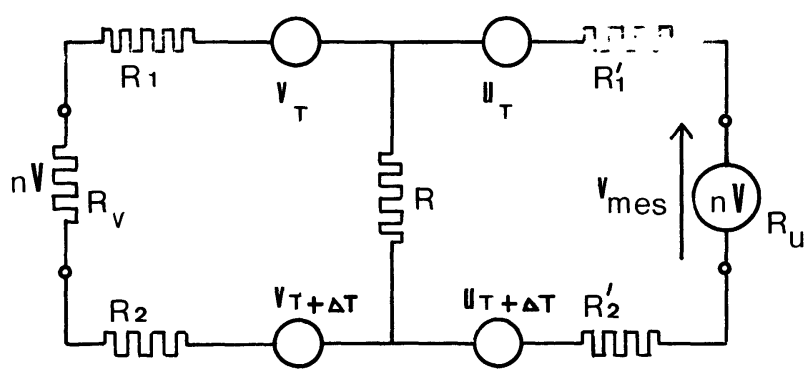

Fig. 6. - Schéma électrique équivalent de l'ensemble échantillon - fils de thermocouple - nanovoltmètres.

[Equivalent circuit for sample-thermocouple wires-nanovoltmeters.]

L'erreur introduite par le branchement simultané des appareils de mesure reste inférieure à $1 \%$ tant que l'impédance de l'échantillon est inférieure à $1 \mathrm{M} \Omega$.

L'expérimentateur intervient pour afficher sur le régulateur la température du point de mesure. Après stabilisation, le premier micro-four est alimenté à courant continu constant, ce qui permet d'établir un gradient de température $\Delta T_{1}$ de quelques dixièmes de degré dans l'échantillon (l'expérience montre que la valeur de ce courant ne dépend pratiquement pas de la température moyenne $\bar{T}$ et il n'est pas besoin de le modifier pour chaque nouveau point de mesure). Le calculateur surveille l'apparition du régime thermique stationnaire en vérifiant l'évolution et la corrélation des couples $\left(V_{1}, U_{1}\right)$ qui sont soumis à différents tests. Ceux-ci consistent à n'accepter les couples de mesure que lorsque les fluctuations sont inférieures aux limites choisies en début d'expérimentation et qui sont plus ou moins sévères selon la grandeur des f.é.m. En pratique, pour les f.é.m. de quelques dizaines de $\mu \mathrm{V}$, la variation de la f.é.m. $\Delta V$ autorisée sur un palier est choisie égale à $0,05 \mu \mathrm{V}$ et la variation de température $\Delta T$ autorisée est égale à $1 / 1000^{e}$ de degré. Le déroulement de ces opérations est visualisé pour l'expérimentateur sur la table traçante (Fig. 5). Les couples $\left(V_{1}, U_{1}\right)$ acceptés sont retenus pour le calcul de la meilleure pente. Après acceptation de cinq couples consécutifs (succès ininterrompu des tests pendant environ $30 \mathrm{~s}$ ), les f.é.m. des températures des deux faces 
de l'échantillon sont mesurées immédiatement et les températures sont calculées d'après la loi polynomiale $(T=f(E))$ mise en mémoire. Le courant continu constant est basculé sur le deuxième microfour; un autre régime thermique stationnaire s'établit, sous la surveillance du calculateur, qui doit répondre à certaines conditions de symétrie $\left(\Delta T_{1} \sim-\Delta T_{2}\right)$ et d'écart par rapport au premier régime $\left|\Delta T_{1}-\Delta T_{2}\right|$ relativement grand, toutes conditions contrôlées également par le calculateur. Après retenue de 5 couples de mesure $\left(V_{2}, U_{2}\right)$ consécutifs, mesures et calcul des températures, la droite de meilleure pente est tracée, le P.T.E. $S(\bar{T})$, la température moyenne $\bar{T}$, les gradients de température $\Delta T_{1}$ et $\Delta T_{2}$, le P.T.E. $S_{\mathrm{B} / \mathrm{A}}(\bar{T})$ sont imprimés. Certains renseignements statistiques sont fournis relatifs à la précision des mesures qui laissent à l'expérimentateur la possibilité de calculer une nouvelle valeur du P.T.E. à l'aide d'un troisième palier de température ; cette nouvelle mesure doit être effectuée lorsqu'il existe un doute sur la stabilité des f.é.m. parasites. La mesure de la résistance de l'échantillon peut être faite immédiatement après le premier palier de température. Une méthode classique avec inversion de courant est utilisée.

La durée moyenne de mesure de $S(\bar{T})$ est environ égale à $10 \mathrm{~min}$. qui représentent le temps moyen d'obtention de deux paliers de température vérifiant les tests du miniordinateur. Chaque valeur de $S(\bar{T})$ est donc obtenue par traitement par le calculateur d'environ 250 mesures de f.é.m. Ce travail effectué en temps réel représente une procédure qui ne pourrait être en aucun cas suivie par un expérimentateur privé de l'assistance de la chaîne d'acquisition de données. De plus, toutes les opérations ayant été schématisées sur table traçante, il y a possibilité d'un examen et d'une critique ultérieurs de chaque point de mesure $S(\bar{T})$.

4. Résultat des mesures. - Le dispositif ainsi réalisé nous a permis de mesurer des pouvoirs thermoélectriques compris entre quelques $\mu \mathrm{V} / \mathrm{K}$ et quelques centaines de $\mu \mathrm{V} / \mathrm{K}$ sur des échantillons de faible dimension (quelques millimètres) dont la résistance peut atteindre quelques $\mathrm{M} \Omega$, dans un domaine de température compris entre $80 \mathrm{~K}$ et $800 \mathrm{~K}$. Pour un même échantillon et dans les mêmes conditions expérimentales, l'écart entre deux manipulations successives est de l'ordre de $0,5 \%$ pour $S \sim 500 \mu \mathrm{V} / \mathrm{K}$.

L'appareillage a d'abord été testé sur un petit disque d'épaisseur $2 \mathrm{~mm}$ de fer électrolytique prélevé sur un barreau étalon, référence standard SRM $734 \mathrm{~S}$ $(\varnothing=0,64 \mathrm{~cm}$, longueur $30 \mathrm{~cm})$ fourni par le « National Bureau of Standards ". Le domaine de température exploré s'étend de $80 \mathrm{~K}$ à $700 \mathrm{~K}$. Les courbes de la figure 7 reproduisent les résultats obtenus par Hust [7] et certifiés par le « N.B.S. », sur un barreau SRM 734 S, dans des conditions optimum de manipulation, entre 80 et $300 \mathrm{~K}$. Nos résultats (Fig. 7) sont en très bon accord quantitatif avec ceux obtenus par cet auteur : les écarts entre les résultats restent inférieurs à $2 \%$. Les résultats de Hust ont été complétés par Fulkerson (Fig. 7) sur un échantillon $(\varnothing=0,38 \mathrm{~cm}$-longueur $7,68 \mathrm{~cm}$ ) d'origine toutefois différente (ingot iron)

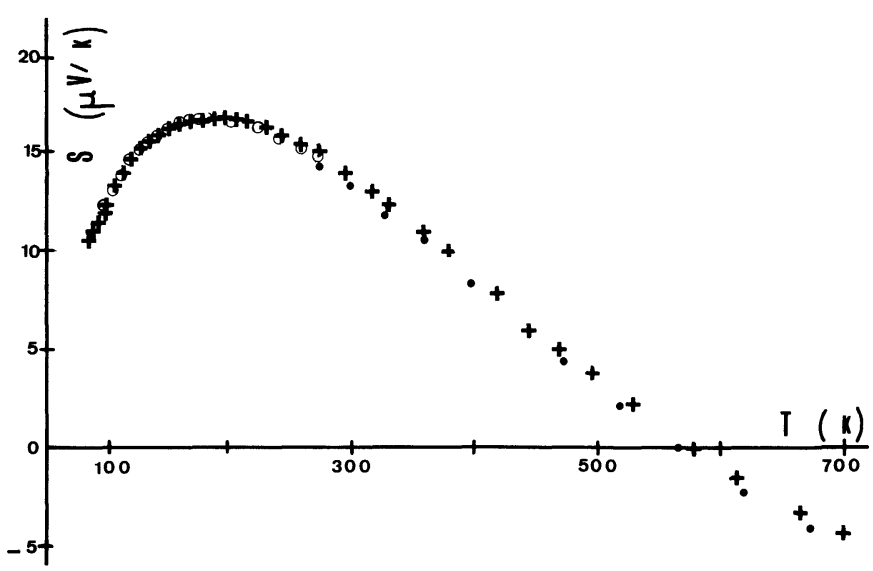

Fig. 7. - Pouvoir thermoélectrique absolu du fer électrolytique étalon entre 80 et $700 \mathrm{~K}$. O Résultats de J. G. Hust et P. Sparks (1975) - SRM 734 S. @ Résultats de W. Fulkerson et al. (1966) - ingot iron. + Nos résultats SRM 734 S.

[Absolute thermoelectric power of standard electrolytic iron : O J. G. Hust and P. Sparks results (1975) SRM 734 S. - W. Fulkerson and al. results (1966) - ingot iron. + Our results - SRM 734 S.]

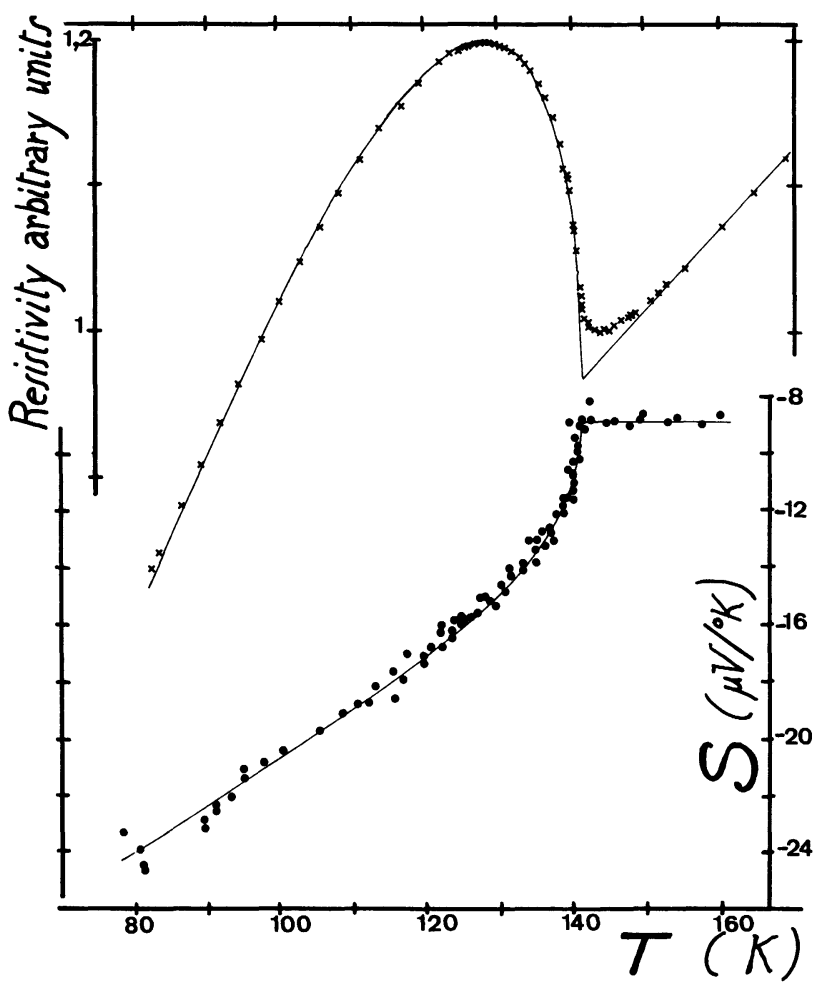

Fig. 8. - Résistivité et pouvoir thermoélectrique de $\mathrm{NbSe}_{3}$ entre 80 et $160 \mathrm{~K}$.

$\left[\mathrm{NBSe}_{3}\right.$ resistivity and T.E.P. in the temperature range $80-160 \mathrm{~K}$. 
entre 300 et $1000 \mathrm{~K}$. Les écarts entre nos résultats et ceux de Fulkerson sont significatifs de la différence de nature des échantillons étudiés.

Afin d'illustrer la reproductibilité de nos mesures, la figure 8 présente les résultats de mesure de P.T.E. obtenus sur $\mathrm{NbSe}_{3}$ au voisinage du point de transition (instabilité structurale) à $144 \mathrm{~K}$ [9]. La faible valeur associée à la variation très abrupte du P.T.E. conduisent à des mesures difficiles à mener et en général peu reproductibles. Notre effort a essentiellement porté sur la détermination précise du comportement du P.T.E. au voisinage du seuil, en multipliant les points de mesure (tous les $1 / 3$ de degré). Nous obtenons des résultats bien reproductibles $(1 \mu \mathrm{V} / \mathrm{K})$ qui confirment l'existence d'une transition du $2 \mathrm{~d}$ ordre.

Par ailleurs, les mesures du P.T.E. et de la conductivité électrique nous ont permis de caractériser les propriétés de $\mathrm{MoTe}_{2}$ monocristallin [10] préparé par la méthode de transport en phase gazeuse (agent de transport : brome) et des composés $\mathrm{MoTe}_{2-x}[11,12]$ $(0<x<0,05)$ appauvris en tellure. Cette méthode de caractérisation s'est révélée très fructueuse en raison de la précision des résultats confirmés par d'autres mesures (optique et diffraction $\mathrm{X}$ ).

\section{Bibliographie}

[1] Conan, A., Goureaux, G. and Zoaeter, M., J. Phys. Chem. Solids 36 (1975) 315.

[2] Arrial, A., Bernede, J. C. et Conan, A., Thin Solid Films 69 (1980) 23.

[3] Dordor, P., Marquestaut, E., Villeneuve, G., Revue Phys. Appl. 15 (1980) 1607.

[4] Conan, A., Goureaux, G., Zoaeter, M., Revue Phys. Appl. 6 (1971) 383.

[5] Casquey, G. R., Sellmyer, D. J., Rubin, L. G., Rev. Sci. Instrum. 40 (1969) 1280.

[6] Rowe, V. A., Schroeder, P., J. Phys. Chem. Solids 31 (1970) 1.

[7] Hust, J. G., Sparks, P., Nat. Bur. Stand., Special publication, 26050 (1975).
[8] Fulkerson, W., Moore, J. P., Mc Elroy, D. L., J. Appl. Phys. 37 (1966) 2639.

[9] Bonnet, A., Conan, A., SAID, P., J. Physique 43 (1982) 675-679.

[10] Conan, A., Delaunay, D., Bonnet, A., Moustafa, A. G., SpIEsser, M., Phys. Status Solidi (b) 94 (1979) 279.

[11] Bonnet, A., Said, P., Conan, A., Rec. Dev. in Cond. Matter Phys. 3 (1981) 36.

[12] Bonnet, A., Conan, A., Saïd, P., 2nd Gen. Conf. of the cond. matter, Division of the EPS, 6A (1982) 419. 